Running Head: MINI-PRFI AND ATTACHMENT

\title{
Costs of Not Getting to Know You: Lower Levels of Parental Reflective Functioning Confer Risk for Maternal Insensitivity and Insecure Infant Attachment
}

\author{
Karin Ensink ${ }^{1}$, Jessica L. Borelli ${ }^{2}$, Julie Roy $^{1}$, Lina Normandin ${ }^{1}$, Arietta Slade ${ }^{3}$, \& Peter Fonagy ${ }^{4}$
}

1. University of Laval

2. University of California, Irvine

3. Yale Child Study Centre

4. University College London

The authors do not have any conflicts of interest to disclose. The authors wish to thank the families who participated in this study. Correspondence regarding this article should be directed to J. Borelli, University of California, Irvine, Department of Psychological Science, 4201 Social and Behavioral Sciences Gateway, Irvine, Ca, 92697, jessica.borelli@uci.edu.A portion of this work was presented by J. Borelli at the conference of the World Association for Infant Mental Health in Rome in 2018. 


\begin{abstract}
Parental reflective functioning $(\mathrm{PRF})$ is a robust predictor of parenting sensitivity and secure infant attachment, but its assessment requires extensive resources, limiting its integration into research and clinical practice. The Mini-Parent Reflective Functioning Interview (Mini-PRFI) assesses the parent's capacity to mentalize for his/her 6 month old infant (rated using the PRF coding system; Slade et al., 2004). In the current study we examined Mini-PRFI scores were associated with theoretically-related constructs; to establish a point of comparison, we evaluated links between Mini-PRFI scores alongside RF assessed from the Adult Attachment Interview (AAI). Mother-infant dyads $(N=88)$ completed the AAI before the birth of the infant, the MiniPRFI and an interaction task (rated for insensitive) when infants were 6 months old, as well as the Strange Situation Procedure when infants were 16 months old. Mini-PRFI scores were strongly positively associated with AAI RF and negatively associated with maternal insensitivity. Mini-PRFI scores predicted infant attachment organization (secure/insecure, organized/disorganized) at 16 months, and this effect was mediated by parenting insensitivity. These findings suggest that the Mini-PRFI predicts theoretically-related attachment constructs, demonstrating the promise of the Mini-PRFI to increase the accessibility of interview-based PRF measurements to clinicians and researchers.
\end{abstract}

Keywords: reflective functioning, mentalization, attachment, sensitivity, disorganization 
Costs of Not Getting to Know You: Lower Levels of Parental Reflective Functioning Confer

Risk for Maternal Insensitivity and Insecure Infant Attachment

Mentalizing is an imaginative mental activity involved in understanding our own and others' behaviors (Fonagy, Gergely, Jurist, \& Target, 2002), and is arguably most important in the context of interactions in close relationships, particularly those characterized by the evocation of attachment needs (Fonagy \& Allison, 2014). Mentalizing is thought to influence the way we behave towards others, and thereby to have implications for the quality of close relationships. When the actions of others are interpreted from a mentalizing stance, people behave more sensitively (Fonagy \& Target, 1997). Scholars contend that the capacity to mentalize is rooted in the context of early attachment relationships (Fonagy, Gergely, Jurist, \& Target, 2002). Operationalized as reflective functioning (RF) for measurement purposes, studies demonstrate that mentalizing protects against psychopathology in children and adolescents (Ensink et al., 2016), underscoring the importance of understanding its correlates in different relational contexts.

\section{Parental Reflective Functioning as a Crucial Psychological Capacity in Parenting}

Parental reflective functioning (PRF; Slade, 2005) refers to parents' capacity to infer the meaning of children's behavior in light of underlying mental states (e.g, emotions, cognitions, desires). Slade and colleagues (Slade, Bernbach, Grienenberger, Levy, \& Locker, 2004) advanced the field considerably by developing a coding system for assessing PRF that has demonstrated wide utility in both research and clinical contexts, and is currently used as a central outcome measure in a number of clinical research studies involving parents of young children (e.g., Suchman et al., 2017). Using a similar theoretical framework, but different measurement methodology, other leaders in the field have demonstrated that higher levels of parental mind- 
mindedness (Meins, 1997; Meins, Fernyhough, Fradley \& Tuckey, 2001), insight (Oppenheim, Koren-Korie, Dolev, \& Yirmiya, 2012), and embodied mentalization (Shai \& Belsky, 2017) are associated with parent-infant relationship quality, underscoring the utility of these concepts for understanding attachment relationships.

Researchers argue that PRF promotes sensitive responding to infants' signals, which in turn creates infant security in the attachment relationship and the ability to mentalize. By interpreting the infants' behaviors as if they communicate mental states, the infant learns to think of himself in this way (Fonagy et al., 2002; Fonagy \& Target, 2007; Meins, 1997). Scholars conceptualize as inseparable the way the parent views and treats the infant (Fonagy \& Target, 1997; Meins, 1997), so that PRF can be seen as an orientation where there is an interest in the subjectivity of the child that manifests as multiple levels of the interaction (Ensink, LeRoux, Biberdzic, Normandin \& Fonagy, 2017). In contrast, when an infant is parented insensitively, which involves failure to acknowledge or understand the child's mental states, the child develops an insecure attachment, characterized by a lack of confidence in others' responsiveness and availability (Bowlby, 1973; Fonagy et al., 2002). Thus, according to both theory and research, PRF should be robustly associated with indices of parent-infant relationship quality, including parental behavior and infant attachment.

This rich theorizing is supported by research -- parents with higher PRF exhibit greater sensitivity in interactions with their infants, and are more likely to have infants who are securely attached, whereas parents who have low PRF are more likely to parent insensitively and to have children who are insecure or disorganized in relation to attachment (Grienenberger, Kelly, \& Slade, 2005; Slade, Grienenberger, Bernbach, Levy, \& Locker, 2005; Stacks et al., 2014; Suchman et al., 2010). In addition to promoting sensitive parenting, PRF may play an important 
role in inhibiting insensitive parenting by helping parents mentally step back and be cognizant of their own emotion so that they can help the infant regulate his/her arousal (Borelli, Hong, Rasmussen, \& Smiley, 2017; Ensink, Normandin, Plamondon \& Berthelot, 2016).

In contrast to organized forms of infant attachment insecurity (e.g., avoidant, ambivalent/resistant), disorganized attachment constitutes a breakdown in attachment strategy, and is thought to result when a child experiences an approach-avoid conflict in which the parent's behavior frightens the child (Main \& Hesse, 1990). As compared to other forms of attachment, relatively less research has explored the parent-child interactional precursors to disorganized attachment (Beebe \& Steele, 2013). To date, researchers have linked disorganized attachment to extreme forms of parenting insensitivity, such as threatening and fear inducing behaviors (Bernier \& Meins, 2008; Hesse \& Main, 2000; Main \& Hesse, 1990), but also to less extreme forms of insensitivity, such as dissociative, deferential, and hyper-sexualized behaviors (Main \& Hesse, 1990; van IJzendoorn, 1995; Van IJzendoorn \& Bakermans-Kranenburg, 2006), and to affective communication errors, intrusiveness, and withdrawal (Beebe et al., 2010; Bernier \& Meins, 2008; Lyons-Ruth et al., 1999; Lyons-Ruth \& Spielman, 2004 ; Madigan, Moran, \& Pederson, 2006). As described above, disorganized infant attachment has been less well studied in terms of its risk factors, including both parental insensitivity and especially PRF. To date, the link between PRF and lower incidence of disorganized attachment has only been identified in a single study, which found that higher PRF was associated with less disruptive affective communication with children, which in turn predicted infant attachment (Grienenberger et al., 2005). A central goal of this study was to provide the first examination of PRF measured during early infancy (six months of age) as a predictor of both insensitive parenting and attachment (secure/insecure and organized/disorganized). 


\section{Assessment of Parents' Reflective Functioning: An Eye toward Feasibility}

Despite the demonstrated utility of measuring and considering PRF for research and intervention, researchers and clinicians are limited due to the fact that its assessment is a time consuming and resource intensive process. At present, the PRF coding system is primarily used with the Parent Development Interview (PDI: Slade, Aber, Bresgi, Berger, \& Kaplan, 2004), a lengthy interview involving an in-depth examination of the parent's perception of him/herself, the child, and the parent's relationship with the child. The PDI yields rich narrative data, but in terms of resources, this one hour interview, subsequent 4-8 hours of transcription and multiple hours of coding by certified raters requires a substantial investment of time and finances, limiting both its research and clinical use, particularly in low resource contexts. Other systems for assessing mentalization-related constructs involve evaluating parents' statements (Meins et al., 2001) or bodily movements (Shai \& Belsky, 2017) when interacting with infants, or interviewing parents about their infants' thoughts and feelings after they watch videotaped interactions involving themselves and the infants (Koren-Karie, Oppenheim, Dolev, Sher, \& Etzion-Carasso, 2002). These systems may entail less time than the PDI, but still necessitate considerable input in terms of money and time (Meins et al., 1997; Oppenheim et al., 2012; Shai \& Belsky, 2017).

And yet, thinking about the parent's level of RF has important and direct implications for intervention, leading clinicians in under-resourced mental health contexts to request a less time consuming way of assessing PRF that can be integrated into routine practice with parents of infants. There is initial evidence that the PDI coding system can be reliably applied to brief interviews or questions regarding parenting. For instance, it has been used to code parents' responses to questions regarding emotional reactions to a parenting stressor (Borelli, Burkhart, Rasmussen, Brody, \& Sbarra, 2016) and a brief interview after a stressful laboratory task 
completed by the child while the mother observes (Borelli et al., 2017). Further, Luyten and colleagues developed a questionnaire to assess PRF (Luyten, Mayes, Nijssens, \& Fonagy, 2014). As compared to self-reported RF, observed RF offers the advantage of providing a more objective index of behaviour, circumventing limitations caused by reporting biases or lack of insight (Jacobvitz, Curran, \& Moller, 2002; Luyten \& Fonagy, 2014).

According to mentalization theorists, RF has both trait- and state-like qualities (Fonagy et al., 2002). Specifically, RF varies with respect to different relationships (e.g., relationship with one's own parents versus relationship with one's partner versus relationship with one's child) and across different contexts, such as during high and low levels of arousal (e.g., Borelli et al., 2017; Fonagy \& Luyten, 2009). Fonagy grounds this relational specificity hypothesis in the notion that RF is a core psychological capacity that is transferable to different relational contexts, but he stresses that there is an effort involved in this cross-relational translation (Fonagy et al., 2002). Therefore, although we would expect parents' RF with respect to their own childhood relationships with caregivers to be associated with their infants' attachment (Fonagy, Steele, \& Steele, 1991), we would also anticipate finding a greater association between PRF and infant attachment.

Few studies have examined the relationship between RF assessed with respect to different relational contexts (e.g., PRI versus RF regarding one's childhood relationships with caregivers, as assessed on the Adult Attachment Interview, George, Kaplan, \& Main, 1985), which is important in testing Fonagy's theorizing. In the initial validation study of the PDI, Crumbley (2008) found a strong correlation between PDI PRF and AAI RF $(r=0.528)$. Subsequent studies demonstrated that both AAI RF and PDI PRF are associated with more sensitive $(r=.24$, Ensink, Normandin, Plamondon, Berthelot \& Fonagy, 2016; $r=.41$, Rosenblum et al., 2008), and less 
insensitive ( $r=-.48$, Grienenberger et al., 2005; and $r=-.43$, Rosenblum et al., 2008) parental behavior towards infants. Consistent with theory, the correlations between PDI PRF and parental behavior are generally stronger than those observed between AAI RF and parental behavior.

\section{The Current Investigation}

We developed the Mini-PRFI for parents of infants between three and 12 months of age. During the first year of a baby's life, parents confront a particularly steep learning curve at many levels. Amidst sleep deprivation, hormonal changes, and exhaustion, parents are tasked with getting to know and understand beings who are unable to communicate their needs verbally and thus are wholly dependent on the parent's capacity to understand what their behavior communicates about their internal states (Slade, 2005).

In the current study, we administered the AAI to sample of mothers during their pregnancy and the Mini-PRFI when the infants were six months old, at which time mothers also completed an interaction task, from which we assessed maternal insensitivity. When the infants were 16 months old they completed the Strange Situation Procedure (Ainsworth, Blehar, Waters, \& Wall, 1978), which we used to code quality of infants' attachment to their mothers.

We tested four hypotheses. First, we hypothesized that Mini-PRFI scores would be significantly positively associated with AAI RF scores. We chose to compare PRF on the MiniPRFI with RF on the AAI because we wanted to mimic Slade's initial method of developing and testing the PDI (Crumbley, 2008; Slade et al., 2004) and the method employed in the first study linking parent RF to infant attachment (Fonagy et al., 1991).

In testing the remainder of our hypotheses, we conducted the analyses first with MiniPRFI scores and then using AAI RF scores as a source of comparison. Our second hypothesis was that parents with higher PRF would demonstrated lower insensitivity. Third, we anticipated 
that higher PRF would be associated with infant attachment, which we tested in terms of the prediction of secure/insecure and disorganized/organized. This goal was grounded in research suggesting that PRF measured later in infants development predicts infants' attachment (e.g., Grienenberger et al., 2005) as well as studies finding that parents' mentalization-based constructs measured using other coding systems earlier in infants' development (e.g, 6 months of age) predict infant attachment (Arnott \& Meins, 2007; Meins et al., 2001; Shai \& Belsky, 2017).

Finally, we explored a developmental model of the links between PRF, parental behavior, and infant attachment (Fonagy et al., 2002); specifically, we predicted that maternal insensitivity would mediate the longitudinal association between PRF and children's attachment classification (secure/insecure and organized/disorganized). Although research has shown that PRF measured at different points in infants' development predicts infant attachment, the current report constitutes the first of its kind to measure PRF at this developmental stage and the second study using PRF to predict likelihood of disorganized infant attachment.

\section{Method}

\section{Participants}

This study was conducted according to ethical guidelines; the procedures were approved by the Institutional Review Board at University of Laval. Mothers provided written informed consent before any data collection occurred. By posting flyers at community health services, gynecological and obstetric clinics, and commercial outlets for mothers and babies, we recruited pregnant women (age range; $21-43 ; M_{\text {age }}=30.82, S D_{\text {age }}=4.00 ; 64 \%$ primiparous) to participate in a study of the transition from pregnancy to parenthood. We screened interested women via telephone for histories of childhood maltreatment in order to recruit a ratio of approximately $30 \%$ of women exposed to childhood abuse so that the sample would be broadly representative 
of the reported prevalence of childhood maltreatment in the general population (Afifi et al., 2011; Scher, Forde, McQuaid, \& Stein, 2004). Exclusion criteria included age below 19 years, having a high-risk pregnancy or a history of psychosis, drug or alcohol abuse, severe health problems, and illiteracy or intellectual limitations.

From the initial sample participating in the prenatal assessment $(N=115)$, those with complications during the last phases of the pregnancy or with significantly premature infants were later excluded from the study $(n=16)$. Thus, $n=99$ mother-infant dyads participated in the laboratory visit when their infants were 6 months old and $n=88$ participated when the infants were 16 months old, the latter of which constitutes the sample in the current study.

All participants (100\% female caregivers, 50\% male children) were Caucasian, largely reflecting the ethnic composition of the French Canadian city in which the study was conducted. Most participants (66\%) in the sample were in common-law relationships $(32 \%$ married, $2 \%$ single), had a university degree (56\%, 14\% high school diplomas, 30\% some college), and reported annual household incomes between $\$ 60,000$ to $\$ 99,999(47 \% ; 21 \% \$ 30,000$ to $\$ 60,000$, $32 \%$ over $\$ 100,000)$. Thus, although the sample was homogenous in ethnicity and income levels, in terms of levels of child abuse, the sample was representative.

\section{Procedure}

During the third trimester of their pregnancy, women completed the AAI (George et al., 1985) to assess their RF regarding their early attachment experiences. When the infants were 6 months old, mothers returned to the laboratory to complete the Mini-PRFI. Mothers and children completed an interaction task from which insensitive parenting was coded using the DIP scale (Out et al., 2009). When the infants were 16 months old, mothers and infants participated in the Strange Situation Procedure (SSP) (Ainsworth, Blehar, Waters \& Wall, 1978) to assess mother- 
infant attachment. All instruments were coded with non-overlapping teams of coders who were naïve to study hypotheses.

\section{Measures}

Parental reflective functioning. The Mini-PRFI is a semi-structured interview that was designed to assess parent's mentalization about young infants (3-12 months old). The complete text of the interview can be found in this article's online supplemental material; the coding system may be obtained by contacting the first author. To generate the questions included in the Mini-PRFI, the research team, which consists of three experts in the field of RF, as well as the author of the PDI, consulted regarding their combined experiences assessing parental mentalization. These researchers also reviewed existing interview/projective measures of parental representations (e.g., PDI, Working Model of the Child Interview, Zeanah \& Benoit, 1993; AAI). From an initial list of questions generated by the researchers, the team excluded questions thought to be repetitive, unnecessary, or harmful in terms of promoting an open and non-judgmental conversational tone. Piloting of the Mini-PRFI revealed that it lasted 15 minutes on average. The complete text of the interview can be found in this article's online supplemental material.

Mini-PRFI questions are designed to follow a brief play session between parent and infant. It begins with questions that are less threatening in nature (asking the parent about the child's play and interests, then the child's personality). The first 8 questions of the interview pertain to the play session and are intended to tap the parent's capacity to read the infant's interactional cues as a function of mental states. The questions follow an invariant sequence, beginning with a focus on the child's play and what types of play he/she prefers before moving on to how the parent knows when the infant wants to play or stop playing. These questions are 
followed by a second set of 8 questions about the infant as an individual with a distinct temperament/personality. This section begins with a general question about the child's temperament, which can be seen as a way of eliciting implicit RF - meaning, unlike on questions in which the interviewer asks explicitly about the child's mental states, here interviewers provide an opportunity (but not a mandate) for the parent to talk about the child's mind. Note that in order to qualify as mentalizing, the parent's understanding of the child's temperament has to be explicitly linked to mental states. For instance, the parent cannot describe the child as a fussy baby, but rather must clearly state that the child feels irritable, frightened, or uncomfortable. In other words, the parent needs to explicitly link the child's traitlike characteristics (fussiness) and mental states (frightened). This section is followed by more specific questions regarding the parent's capacity to understand the infant's emotions (e.g., infant fear) and behaviors, as well as what they communicate about what the infant wants and needs. Questions also focus on parents' observations regarding infants' reactions to affection from parents versus other adults.

Mini-PRFIs were audio-recorded and transcribed verbatim (see Table 1 for sample excerpts). Interview transcripts were then coded using the PRF Coding system developed by Slade and colleagues (Slade et al., 2004; grounded in Fonagy and colleagues' [1998] original coding system for the AAI). In this coding system, each question receives a score on an 11-point scale (ranging from -1 [anti-reflective] to 9 [exceptional RF]), with higher scores indicating higher RF. Scores lower than three are suggestive of pre-mentalizing. Scores of five and higher denote mentalizing - these responses reflect an understanding of mental states and their influence on behavior. Consistent with the protocol for coding the full-length PDI, on the Mini-PRFI, coders also assign a global PRF score that summarized their RF throughout the interview. To evaluate interrater reliability, the first author and a graduate student in psychology, a trained and reliable rater of RF on the AAI, coded 30 Mini-PRFI transcripts, obtaining an excellent level of interrater 
reliability $I C C(2)=.90, p<.0001$. The graduate student coded the remainder of the interviews. Both coders were naïve to study hypotheses and all other information regarding the participants; the coders of the Mini-PRFI did not code any other measures in the study.

To provide preliminary support of the divergent validity of the measure, we tested whether PRF on the Mini-PRFI was associated with some demographic and psychological variables from which PRF should be distinct. Our analyses revealed that Mini-PRFI was not significantly associated with mothers' self-reported alexithymia, $r=-.03, p=.78$, maternal education, $r=.14, p=.20$, whether the participant was a first time mother, $r=.13, p=.25$, or household income, $r=.16, p=.15$. Similarly, AAI RF was not significantly associated with any of these variables.

Reflective functioning regarding childhood experiences. RF was rated from transcribed AAIs (George et al., 1985) administered during pregnancy. The AAI is a semistructured interview designed to elicit the participant's current state of mind regarding attachment experiences with parents and other significant caregivers during childhood. The interview includes questions about the quality of childhood experiences with parents; the participant's responses to experiences of rejection, separation, loss, and trauma during childhood; and the participant's evaluation of the effects of those childhood experiences on his or her current functioning. The AAI was coded using the RF manual (Fonagy, Target, Steele, \& Steele, 1998). The psychometric properties detailed in the manual include high interrater reliability as well as good discriminant validity and predictive validity across a number of samples. RF is rated on a scale of -1 to 9 , with every score representing a different level of mental state explanation. Ratings of -1 indicate an attack on mentalizing. Ratings of 0 indicate a refusal to engage in mentalizing. Ratings of 1 indicate the absence of any recognition of mental states so 
that interpersonal reactions are described only in behavioral terms, or individuals are described only in terms of physical or global characteristics. Ratings of 3 indicate limited ability to identify and acknowledge simple mental states and affects at a very general level, but without understanding how mental states function. Ratings of 5 indicate a basic capacity to consider how mental states influence behavior and perceptions, and are in turn evoked in interpersonal interactions. Higher ratings indicate increasingly full and sophisticated mental state accounts of subjective experience underlying interactions, and ratings of 9 are reserved for exceptional accounts that reflect nuanced understanding of motivations underlying behavior or unusual insights. RF is typically rated based on all AAI questions that explicitly demand an appreciation of mental states (e.g., "Why did your parents behave as they did during your childhood?”). An overall RF score that represents respondents' characteristic level of RF is derived based on the individual scores using a decision algorithm outlined in the manual and that takes into account the frequency of low scores or failures in mentalizing as well as the frequency of high scores. AAI RF coding protocol entails coding RF with respect to the self and with respect to others, which are summarized in the global RF score, the value most commonly used in research.

In the current study, two graduate students rated the AAI protocols for RF. Interrater reliability was assessed on 19 coding protocols and the intraclass correlation coefficient (ICC) was high, $\operatorname{ICC}(2)=.80, p<.0001)$, indicative of satisfactory interrater reliability. These coders were naïve to study hypotheses and all other information regarding the participants and did not code any other measures in the study.

Our analyses revealed that mothers' RF on the AAI was not significantly associated with mothers' self-reported alexithymia, maternal education, whether the participant was a first time mother, or household income. 
Maternal insensitivity. The Disconnected and Extremely Insensitive Parenting (DIP) scale was developed to assess all forms of parental behavior previously linked to disorganized infant attachment, including subtler forms of insensitivity, such as disconnected behavior, as well as more extreme forms of insensitive behavior (Out, Bakermans-Kranenberg, \& Van IJzendoorn, 2009). We use the DIP scale in order to emulate prior research exploring links between PRF and infant attachment as mediated by parental behavior (Grienenberger et al., 2005) ${ }^{1}$. The DIP assesses insensitive behavior on two dimensions - 1) disconnected parenting behavior (Main \& Hesse, 1998) and 2) extreme parental insensitivity (e.g., parental withdrawal and neglect, and intrusive, negative, aggressive, or otherwise harsh parental behaviors; Lyons-Ruth et al., 1999; Out et al., 2009). These two dimensions can be averaged to create a mean insensitivity score. The DIP scale has been shown to have good psychometric properties (Out et al., 2009).

In the DIP scale, discrete disconnected and extremely insensitive behaviors are rated on a 9-point scale each time they occur. For both dimensions, a final score is assigned, equal to the highest individual score or 1 point higher if the behavior was severe or occurred frequently. In the present study, the DIP scale was rated by two graduate students who were trained by the developers of the measure, and the ICC for the global scores was high, disconnected: ICC(2)= .92 ) and extreme insensitivity: $I C C(2)=.79)$. The coders were naïve to study hypotheses and all other information regarding the participants; the coders of the DIP did not code any other measures in the study.

Infant attachment. The Strange Situation Procedure (SSP; Ainsworth et al., 1978) was used to assess infant attachment organization when infants were approximately 16 months old.

\footnotetext{
${ }^{1}$ We note that in this investigation we also assessed maternal sensitivity using the Ainsworth scoring procedures; the results of the sensitivity operated similarly as the insensitivity DIP scale. However, due to our desire to replicate prior work (Grienenberger et al., 2005), as well as our desire to predict disorganized attachment specifically, here we exclusively present the analyses using the DIP scale. DIP and maternal sensitivity using the Ainsworth scale were inversely associated, $r=-.32, p=002$.
} 
The SSP is widely used and its validity has been demonstrated in major long-term studies (Sroufe, Egeland, Carlson, \& Collins, 2005). It consists of eight 3-min episodes during which the mother leaves (separation episodes) and reunites with the infant (reunion episodes). Two trained coders rated SSPs for the three organized attachment classifications (secure, anxious-resistantinsecure, and anxious-avoidant- insecure) using the criteria of Ainsworth et al. (1978), and attachment disorganization using the criteria of Main and Solomon (1990). All coders were naïve to study hypotheses and all other information regarding the participants; the coders of the SSP did not code any other measures in the study. In this sample, 50\% were double coded with near full agreement for the organized attachment classifications, and 97\% agreement for infant attachment disorganization.

\section{Data Analytic Plan}

A set of preliminary analyses revealed that mothers' abuse history was not significantly associated with any of the key study variables; therefore, we did not include abuse history as a control variable. We tested hypotheses involving continuous dependent variables using hierarchical linear regressions, controlling for variables that were significantly associated with any of the three dependent variables in an initial step. We tested the hypotheses involving infants' attachment classification (the dichotomous categorical secure/insecure of organized/disorganized variables) using logistic regression. To test hypotheses involving mediation, we used Model 4 of Hayes' PROCESS Macro for SPSS (see Hayes, 2013, which tests all paths simultaneously using bootstrapping to estimate indirect effects and corresponding 95\% confidence intervals (CIs). PROCESS Model 4 generates estimates of indirect effects, direct effects, and total effects for each mediation model, which surpass a statistical significance threshold of $p<.05$ when the $95 \%$ CI does not include zero. Due to the fact that we were 
interested in identifying whether mothers' Mini-PRFI scores performed comparably to AAI RF, we conducted all analyses first using Mini-PRFI and then using AAI RF.

\section{Results}

The majority of infants were classified as secure $(n=48,54.5 \%)$ in the SSP, with 14 classified as avoidant (15.9\%), 10 as ambivalent-resistant (11.4\%), and 16 as disorganized (18.2\%). Zero-order correlations suggested that male children were more likely to be classified as disorganized, $r=.25, p=.02$ (see Table 2). Mini-PRFI was positively associated with AAI RF $\left(r_{\text {global }}=.51, p_{\text {global }}<.0001 ; r_{\text {self }}=.37, p_{\text {self }}=.001 ; r_{\text {other }}=.46, p_{\text {other }}<.0001\right)$, and was negatively associated with maternal insensitivity, $r=-.24, p=.03$, and disorganized infant attachment, $r=$ $.34, p=.002$. Mothers with abuse histories did not have significantly lower Mini-PRFI, $p=.07$, or AAI RF, $p=.19$, than mothers without abuse histories.

\section{Hypothesis 1: Is PRF on the Mini-PRFI positively associated with RF on the AAI?}

The results of the bivariate correlations described above (see also Table 2) confirmed that Mini-PRFI scores were significantly positively associated with AAI RF (global, self, and other). Notably, Mini-PRFI scores were most strongly associated with AAI global RF scores, which is the AAI RF scale most commonly used in research studies.

\section{Hypothesis 2: Are Mini-PRFI scores concurrently associated with maternal}

insensitivity? The results of a hierarchical linear regression revealed that after controlling for child sex, $\mathrm{R}^{2}=.01, p=.81$, Mini-PRFI scores were a significant predictor of insensitivity, $\Delta \mathrm{R}^{2}=$ $.02, p=.04, \beta=-.24,95 \%$ CI $[-.80,-.03]-$ higher PRF was linked to lower insensitivity.

Second, controlling for infant sex, AAI RF was not a significant predictor of insensitivity, $\Delta \mathrm{R}^{2}=.04, p=.07, \beta=-.20,95 \%$ CI $[-.83, .03]$.

Hypothesis 3: Are Mini-PRFI scores prospectively predictive of infant attachment? 
First we tested whether RF scores predicted secure versus insecure attachment.

Controlling for child sex on an initial step, Wald $\chi^{2}(1,83)=5.11, p=.08$, a binary logistic regression revealed that Mini-PRFI RF scores significantly altered the odds ratio in the prediction of infant attachment security, Wald $\chi^{2}(1,83)=3.33, p=.03$. As Mini-PRFI scores increased, the odds of the infant being classified with secure attachment also increased, $\beta=1.36$, $p=.03$ (see Figure 1). In contrast, AAI RF was not a significant predictor of infant attachment security, Wald $\chi^{2}(1,83)=0.59, p=.23$.

Next we tested whether RF predicted disorganized versus organized infant attachment. After controlling for child sex on an initial step, Wald $\chi^{2}(1,83)=5.11, p=.08$, a binary logistic regression revealed that Mini-PRFI scores significantly altered the odds ratio in the prediction of infant disorganized attachment, Wald $\chi^{2}(1,83)=9.47, p=.002$. As Mini-PRFI scores increased, the odds of the infant being classified with disorganized attachment decreased, $\beta=.83, p=.002$ (see Figure 1). As above, AAI RF was not a significant predictor of disorganized attachment.

\section{Hypothesis 4: Does maternal insensitivity mediate the prospective association}

between Mini-PRFI scores and children's attachment? After controlling for child sex, a regression showed that maternal insensitivity had a significant indirect effect in the model examining the association between Mini-PRFI RF and children's attachment security, $b=.10$, $\mathrm{SE}=.07,95 \% \mathrm{CI}[.004, .28]$. Similarly, after controlling for child sex, maternal insensitivity had a significant indirect effect in the model examining the association between Mini-PRFI RF and children's disorganized attachment, $b=.17, \mathrm{SE}=.14,95 \% \mathrm{CI}[.02, .51]$ (see Figure 2).

We did not test AAI RF as mediator since the analyses involving the individual pathways were not statistically significant.

\section{Discussion}


The aims of this study were both methodological and conceptual in nature. The methodological aim entailed conducting a preliminary exploration of the association between Mini-PRFI scores AAI RF scores. In addition, we sought to provide the first examination of PRF during this age range of infancy and the second examination of the links between PRF with insensitivity and infant attachment.

In support of hypotheses, Mini-PRFI scores correlated positively with AAI RF scores. Importantly, the strength of the relationship $(r=.51)$ was similar to that previously reported between the PDI and AAI ( $r=0.53$; Crumbley, 2008). The strong positive correlation between these two measures of RF support Fonagy's theorizing that mentalizing is a core psychological capacity transferrable to different relational contexts (Fonagy et al., 2002), though importantly, the two measures of RF are not redundant.

With regard to the conceptual aim of the study, the findings revealed that Mini-PRFI scores were a significant predictor of insensitivity, with better PRF associated with fewer negative parenting behaviors. Furthermore, Mini-PRFI also prospectively predicted infant attachment - as Mini-PRFI scores increased, the odds of infants being classified as having secure (as opposed to insecure) and organized (as opposed to disorganized) attachment also increased. Insensitivity indirectly linked Mini-PRFI scores and infant attachment, after controlling for child sex. Importantly, our test of indirect effects should be evaluated in the context of knowing that two of the measures (mediator and independent variable) were assessed at the same time. Nonetheless, we believe that the findings provide promising initial evidence that a mother's understanding of her infant may have important implications for later infant attachment organization, as explained through their mutual association with maternal insensitivity. Perhaps a parent's capacity to view the infant as an intentional agent influences the capacity to inhibit 
negative behaviors in interaction with the infant, which in turn predicts infant attachment.

Mentalizing about the impact of our behavior on others inhibits responding insensitively because awareness of the distress, hurt and fear that this evokes serve as a brake that facilitates selfregulation and behavioural control (Ensink, Normandin, Berthelot, Plamandon \& Fonagy, 2016). While mentalizing has been shown to underlie sensitive responding to the infant's needs and to be associated with infant attachment security, it is insensitive responding that is most likely to undermine the development of organized attachment strategies and thus have important implications for self organization.

In sum, the findings of the present study show that mothers' capacity to mentalize about their infants has important longitudinal implications for infant attachment organization. This finding adds to the literature regarding the importance of RF in general for attachment relationships as well as the importance of PRF for the quality of parent child relationships. As Fonagy and Allison (2014) suggest, RF is likely most important when taking care of infants and young children, in which interactions are characterized by high levels of dependency and intense emotions, demanding a more nuanced understanding of self and other than is normally required. We know from previous research that other dimensions of RF, for example, RF regarding childhood experiences of relational trauma (Berthelot, Ensink, Bernazzani, Normandin, Luyten \& Fonagy, 2015), RF regarding self (Suchman, DeCoste, Leigh, \& Borelli, 2010), and RF regarding school-aged children (Borelli et al., 2015; Borelli, Hong, et al. 2017, Ensink et al., 2014) also have important implications for the quality of parent child relationships and child adaptation, depending on the specific population studied and which interpersonal issues are likely to be the most challenging. In any study we are usually at best able to consider one or two dimensions of RF, and while it is important to highlight the implications of specific dimensions 
of mentalization, it is also crucial to remember that there are probably many different dimensions of mentalization that contribute to adaptive functioning at a personal and interpersonal level.

In sum, we contend that the results of this study suggest initial promise of the Mini-PRFI as a tool that could enhance the accessibility of the PRF construct to lower-resource research and clinical contexts. High levels of inter-rater reliability were obtained when using the PDI coding system to code the Mini-PRFI; these were comparable in magnitude with respect to raters' reliability on AAI RF. Thus, with limited training, raters who have previously demonstrated reliability as coders of RF on the PDI or AAI RF can be trained to code the Mini-PRFI reliably. The next step in this line of work will be to explore whether clinicians or researchers who have not previously been trained in RF coding can beceom reliable coders of the Mini-PRFI.

\section{Strengths and Limitations}

While the study has many strengths, including its longitudinal design and the inclusion of observational measures of RF, parental behavior, and infant attachment, the findings need to be interpreted in light of certain limitations. We note that since this study did not involve a comprehensive evaluation of validity and reliability of the Mini-PRFI, the conclusion that the measure has adequate reliability and validity is premature. For instance, the range of variables examined for divergent validity was limited, underscoring the need for extension of these findings. In addition, in this study we chose to utilize the AAI as the point of comparison for the Mini-PRFI for two reasons - first, we sought to maintain consistency with Slade's initial way of establishing the PDI as a measure of RF, which she did by using the RF coding system for the AAI (Crumbley, 2008; Slade et al., 2004); and second, we wanted to attempt to replicate the first study linking RF to infant attachment (Fonagy et al., 1991). However, in future studies, it will be important to directly compare Mini-PRFI to the PDI, in terms of both their intercorrelations and 
their respective predictive validity. This information regarding the relative advantages of the two measures will enable researchers to make well-informed decisions regarding resource allocation. In addition, it is also a limitation that the Mini-PRFI coders used in the current study had previously been certified as reliable coders of the PDI; another important step in this line of work is to evaluate whether coders who exclusively have received training in coding the Mini-PRFI can become reliable coders of this interview. Further, the two measures of RF were obtained at different time points (AAI during pregnancy, Mini-PRFI six months post-delivery). Although the high correlation between the two measures is impressive evidence of the longitudinal stability of $\mathrm{RF}$, in order to more precisely characterize the construct validity of the Mini-PRFI, administering the two interviews at the same timepoint would have been preferable. That the Mini-PRFI was administered closer to the assessment of relevant outcomes may in part account for its stronger associations with these variables. In addition, we note that shared method variance may at least in part have contributed to the association between Mini-PRFI and AAI RF scores. It is also worth considering that although a portion of all measures were double-coded with adequate levels of inter-rater reliability, we did not double-code all assessments for all participants in the investigation, which leaves open a possibility of coder bias.

Further, though the sample size is considered typical for this type of study, it may have limited the capacity to detect significance of effects. And though longitudinal, the design was correlational, precluding causal inferences, which prevents us from testing mechanistic models. Finally, the sample was racially homogeneous and predominantly comprised of middle-SES and educated mothers, suggesting the need for additional studies using the Mini-PRFI among more diverse samples. Limitations notwithstanding, our hope is that this study will lay the necessary groundwork for future use of the Mini-PRFI in research and clinical contexts, inspiring further 
validity studies and increasing the accessibility of the assessment of a key construct within attachment research.

\section{Conclusion}

The findings of the study confirm that it is possible to rate PRF reliably from the MiniPRFI, a brief interview that assesses parents' capacity to mentalize about their young infants. Mothers' RF about the their six months old infants predicted infant attachment organization at 16 months, and this relationship was indirectly linked through a mutual association with parenting insensitivity. These findings provide evidence that PRF measured as early as six months of age prospectively predicts both parent and child outomes at 16 months. 


\section{References}

Afifi, T. O., Mather, A., Boman, J., Fleisher, W., Enns, M. W., Macmillan, H., \& Sareen, J. (2011). Childhood adversity and personality disorders: results from a nationally representative population-based study. Journal of Psychiatric Research, 45, 814-822. https://doi.org/10.1016/j.jpsychires.2010.11.008

Ainsworth, M. D. S., Blehar, M. C., Waters, E., \& Wall, S. (1978). Patterns of Attachment: A Psychological Study of the Strange Situation. Hillsdale: NJ: Erlbaum.

Arnott, B., \& Meins, E. (2007). Links among antenatal attachment representations, postnatal mind-mindedness, and infant attachment security: A preliminary study of mothers and fathers. Bulletin of the Meninger Clinic, 71, 132-149. doi: 10.1521/bumc.2007.71.2.132

Bakermans-Kranenburg, M. J., \& van IJzendoorn, M. H. (2009). The first 10,000 Adult Attachment Interviews: distributions of adult attachment representations in clinical and non-clinical groups. Attachment \& Human Development, 11, 223-263. https://doi.org/10.1080/14616730902814762

Beebe, B., Jaffe, J., Markese, S., Buck, K., Chen, H., Cohen, P., ... Feldstein, S. (2010). The origins of 12-month attachment: a microanalysis of 4-month mother-infant interaction. Attachment \& Human Development, 12(1-2), 3-141. https://doi.org/10.1080/14616730903338985

Beebe, B., \& Steele, M. (2013). How Does Microanalysis of Mother-Infant Communication Inform Maternal Sensitivity and Infant Attachment? Attachment \& Human Development, 15. https://doi.org/10.1080/14616734.2013.841050

Bernier, A., \& Meins, E. (2008). A threshold approach to understanding the origins of attachment disorganization. Developmental Psychology, 44, 969-982. 
https://doi.org/10.1037/0012-1649.44.4.969

Berthelot, N., Ensink, K., Bernazzani, O., Normandin, L., Luyten, P., \& Fonagy, P. (2015). Intergenerational Transmission of Attachment in Abused and Neglected Mothers: The Role of Trauma-Specific Reflective Functioning. Infant Mental Health Journal, 36, 200212. https://doi.org/10.1002/imhj.21499

Borelli, J. L., Burkhart, M. L., Rasmussen, H. F., Brody, R., \& Sbarra, D. A. (2017). Secure Base Script Content Explains the Association Between Attachment Avoidance and EmotionRelated Constructs in Parents of Young Children. Infant Mental Health Journal, 38, 210225. https://doi.org/10.1002/imhj.21632

Borelli, J. L., Hong, K., Rasmussen, H. F., \& Smiley, P. A. (2017). Reflective Functioning, Physiological Reactivity, and Overcontrol in Mothers: Links With School-Aged Children's Reflective Functioning. Developmental Psychology. https://doi.org/10.1037/dev0000371

Borelli, J. L., St John, H. K., Cho, E., \& Suchman, N. E. (2016). Reflective functioning in parents of school-aged children. The American Journal of Orthopsychiatry, 86, 24-36. https://doi.org/10.1037/ort0000141

Ensink, K., Bégin, M., Normandin, L., \& Fonagy, P. (2016). Maternal and child reflective functioning in the context of child sexual abuse: pathways to depression and externalising difficulties. European Journal of Psychotraumatology, 7, 30611. https://doi.org/10.3402/ejpt.v7.30611

Ensink, K., Leroux, A., Normandin, L., Biberdzic, M., \& Fonagy, P. (2017). Assessing Reflective Parenting in Interaction With School-Aged Children. Journal of Personality Assessment, 1-11. https://doi.org/10.1080/00223891.2016.1270289 
Ensink, K., Normandin, L., Plamondon, A., Berthelot, N., \& Fonagy, P. (2016). Intergenerational pathways from reflective functioning to infant attachment through parenting. Canadian Journal of Behavioural Science / Revue Canadienne Des Sciences Du Comportement, 48, 9-18. https://doi.org/10.1037/cbs0000030

Ensink, K., Normandin, L., Target, M., Fonagy, P., Sabourin, S., \& Berthelot, N. (2014). Mentalization in children and mothers in the context of trauma: An initial study of the validity of the Child Reflective Functioning Scale. British Journal of Developmental Psychology, 33, 203-217. https://doi.org/10.1111/bjdp.12074

Fonagy, P., \& Allison, E. (2014). The role of mentalizing and epistemic trust in the therapeutic relationship. Psychotherapy (Chicago, Ill.), 51, 372-380. https://doi.org/10.1037/a0036505

Fonagy, P., Gergely, G., Jurist, E., \& Target, M. (2002). Affect Regulation, Mentalization, and the Development of Self (1 edition). New York: Other Press.

Fonagy, P., \& Luyten, P. (2009). A developmental, mentalization-based approach to the understanding and treatment of borderline personality disorder. Development and Psychopathology, 21, 1355-1381. https://doi.org/10.1017/S0954579409990198

Fonagy, P., Steele, H., \& Steele, M. (1991). Maternal representations of attachment during pregnancy predict the organization of infant-mother attachment at one year of age. Child Development, 62, 891-905.

Fonagy, P., \& Target, M. (1997). Attachment and reflective function: their role in selforganization. Development and Psychopathology, 9, 679-700.

Fonagy, P., \& Target, M. (2007). Playing with reality. The International Journal of Psychoanalysis, 88, 917-937. https://doi.org/10.1516/4774-6173-241T-7225 
Fonagy, P., Target, M., Steele, H., \& Steele, M. (1998). Reflective-functioning manual, version 5.0, for application to adult attachment interviews. London: University College London.

George, C., Kaplan, N., \& Main, M. (1985). The Adult Attachment Interview. Unpublished manuscript. Department of Psychology, University of California, Berkeley, CA.

Grienenberger, J. F., Kelly, K., \& Slade, A. (2005). Maternal reflective functioning, motherinfant affective communication, and infant attachment: exploring the link between mental states and observed caregiving behavior in the intergenerational transmission of attachment. Attachment \& Human Development, 7, 299-311.

https://doi.org/10.1080/14616730500245963

Hesse, E., \& Main, M. (2000). Disorganized infant, child, and adult attachment: collapse in behavioral and attentional strategies. Journal of the American Psychoanalytic Association, 48, 1097-1127; discussion 1175-1187. https://doi.org/10.1177/00030651000480041101

Jacobvitz, D., Curran, M., \& Moller, N. (2002). Measurement of adult attachment: the place of self-report and interview methodologies. Attachment \& Human Development, 4, 207215.

Koren-Karie, N., Oppenheim, D., Dolev, S., Sher, E., \& Etzan-Carasso, A. (2002). Mothers' insightfulness regarding their infants' internal experience: Relations with maternal sensitivity and infant attachment. Developmental Psychology, 38(4), 534-542. http://dx.doi.org/10.1037/0012-1649.38.4.534

Luyten, P., \& Fonagy, P. (2014). The Routledge Handbook of Attachment: Assessment. In The Routledge Handbook of Attachment: Assessment. Routledge.

Luyten, P., Mayes, L. C., Nijssens, L., \& Fonagy, P. (2017). The parental reflective functioning 
questionnaire: Development and preliminary validation. PLOS ONE, 12, e0176218. https://doi.org/10.1371/journal.pone.0176218

Lyons-Ruth, K., Bronfman, E., \& Parsons, E. (1999). Atypical attachment in infancy and early childhood among children at developmental risk. IV. Maternal frightened, frightening, or atypical behavior and disorganized infant attachment patterns. Monographs of the Society for Research in Child Development, 64(3), 67-96; discussion 213-220.

Lyons-Ruth, K., \& Spielman, E. (2004). Disorganized Infant Attachment Strategies And Helpless-Fearful Profiles Of Parenting: Integrating Attachment Research With Clinical Intervention. Infant Mental Health Journal, 25, 318-335. https://doi.org/10.1002/imhj.20008

Madigan, S., Moran, G., \& Pederson, D. R. (2006). Unresolved states of mind, disorganized attachment relationships, and disrupted interactions of adolescent mothers and their infants. Developmental Psychology, 42, 293-304. https://doi.org/10.1037/00121649.42 .2 .293

Main, M., \& Hesse, E. (1990). Parents' unresolved traumatic experiences are related to infant disorganized attachment status: Is frightened and/or frightening parental behavior the linking mechanism? In The John D. and Catherine T. MacArthur Foundation series on mental health and development. Attachment in the preschool years: Theory, research, and intervention (pp. 161-182). Chicago: University of Chicago Press.

Main, M., \& Hesse, E. (1998). Frightening, frightened, dissociated, deferential, sexualized and disorganized parental behavior: A coding system for parent-infant interactions (6th ed). Unpublished manual. University of California at Berkeley.

Meins, E. (1997). Security of attachment and maternal tutoring strategies: Interaction within the 
zone of proximal development. British Journal of Developmental Psychology, 15, 129144. https://doi.org/10.1111/j.2044-835X.1997.tb00730.x

Meins, E., Fernyhough, C., Fradley, E., \& Tuckey, M. (2001). Rethinking maternal sensitivity: mothers' comments on infants' mental processes predict security of attachment at 12 months. Journal of Child Psychology and Psychiatry, and Allied Disciplines, 42, 637648.

Oppenheim, D., Koren-Karie, N., Dolev, S., \& Yirmiya, N. (2012). Maternal sensitivity mediates the link between maternal insightfulness/resolution and child-mother attachment: the case of children with Autism Spectrum Disorder. Attachment \& Human Development, 14, 567-584. https://doi.org/10.1080/14616734.2012.727256

Out, D., Bakermans-Kranenburg, M. J., \& IJzendoorn, M. H. V. (2009). The role of disconnected and extremely insensitive parenting in the development of disorganized attachment: validation of a new measure. Attachment \& Human Development, 11, 419-443. https://doi.org/10.1080/14616730903132289

Rosenblum, K. L., McDonough, S. C., Sameroff, A. J., \& Muzik, M. (2008). Reflection in thought and action: Maternal parenting reflectivity predicts mind-minded comments and interactive behavior. Infant Mental Health Journal, 29, 362-376. https://doi.org/10.1002/imhj.20184

Scher, C. D., Forde, D. R., McQuaid, J. R., \& Stein, M. B. (2004). Prevalence and demographic correlates of childhood maltreatment in an adult community sample. Child Abuse \& Neglect, 28, 167-180. https://doi.org/10.1016/j.chiabu.2003.09.012

Shai, D., \& Belsky, J. (2017). Parental embodied mentalizing: how the nonverbal dance between parents and infants predicts children's socio-emotional functioning. Attachment and 
Human Development, 2, doi:10.1080/14616730500245963

Slade, A. (2005). Parental reflective functioning: an introduction. Attachment \& Human Development, 7, 269-281. https://doi.org/10.1080/14616730500245906

Slade, A., Aber, J. L., Bresgi, I., Berger, B., \& Kaplan. (2004). The parent development interview Revised. Unpublished protocol. New York, NY: The City University of New York.

Slade, A., Grienenberger, J., Bernbach, E., Levy, D., \& Locker, A. (2005). Maternal reflective functioning, attachment, and the transmission gap: A preliminary study. Attachment \& Human Development, 7, 283-298. https://doi.org/10.1080/14616730500245880

Sroufe, L. A., Egeland, B., Carlson, E., \& Collins, W. A. (2005). The development of the person: The Minnesota study of risk and adaptation from birth to adulthood. New York: Guilford.

Stacks, A. M., Muzik, M., Wong, K., Beeghly, M., Huth-Bocks, A., Irwin, J. L., \& Rosenblum, K. L. (2014). Maternal Reflective Functioning among Mothers with Childhood Maltreatment Histories: Links to Sensitive Parenting and Infant Attachment Security. Attachment \& Human Development, 16, 515-533. https://doi.org/10.1080/14616734.2014.935452

Suchman, N. E., DeCoste, C. L., McMahon, T. J., Dalton, R., Mayes, L. C., \& Borelli, J. (2017). Mothering From the Inside Out: Results of a Second Randomized Clinical Trial Testing a Mentalization-Based Intervention for Mothers in Addiction Treatment. Development and Psychopathology, 29, 617-636. https://doi.org/10.1017/S0954579417000220

Suchman, N. E., DeCoste, C., Leigh, D., \& Borelli, J. (2010). Reflective functioning in mothers with drug use disorders: implications for dyadic interactions with infants and toddlers. Attachment \& Human Development, 12, 567-585. 
https://doi.org/10.1080/14616734.2010.501988

Van IJzendoorn, M. H., \& Zwart-Woudstra, H. A. (1995). Adolescents' attachment representations and moral reasoning. The Journal of Genetic Psychology, 156(3), 359372. https://doi.org/10.1080/00221325.1995.9914829

Van IJzendoorn, Marinus H., \& Bakermans-Kranenburg, M. J. (2006). DRD4 7-repeat polymorphism moderates the association between maternal unresolved loss or trauma and infant disorganization. Attachment \& Human Development, 8, 291-307. https://doi.org/10.1080/14616730601048159 
Table 1.

Excerpts of responses to the Mini-PRFI.

\begin{tabular}{|c|c|}
\hline Score & Interview Excerpt \\
\hline 0 & How would you describe her child? I don't know. \\
\hline 1 & $\begin{array}{l}\text { How would you describe your child? He is smiling. Because I have a photo of him when he was born and he had a little smile. I put it on } \\
\text { the wall of his bedroom. How does he respond when he is left alone on his own? Well I can't say. He does not notice. } \\
\text { Does she have a favorite toy? Well, there are thousands of toys and things at our place, so I don't know. How do you know that she wants } \\
\text { to stop playing? It is determined by our routine, when it is time to stop, it is time. }\end{array}$ \\
\hline 2 & How do you know that she is hungry? Babies have a special cry when they are hungry. \\
\hline 3 & $\begin{array}{l}\text { How do you know that she wants to stop playing? When it has been a while that I have played with him and he seems to find nothing } \\
\text { interesting or funny and just cries. } \\
\text { How does he respond when he is left alone on his own? At first he does not notice, and he just continues playing, and then when he does he } \\
\text { starts crying. }\end{array}$ \\
\hline 4 & $\begin{array}{l}\text { How does he respond when he is left alone on his own? He plays alone, but then I don't know why sometimes he does not want to stay } \\
\text { calmly alone in the lounge. Then he calls and we go and see. But when he has slept and eaten well I put him in the lounge and he can } \\
\text { play alone. }\end{array}$ \\
\hline 5 & $\begin{array}{l}\text { With my partner, it is more difficult. I am not there of course so have not really seen this but probably my partner is more stressed to } \\
\text { know how she will be and how she feels. She cries more and is inconsolable. It is not easy. }\end{array}$ \\
\hline 6 & $\begin{array}{l}\text { How would you describe your child? He is not a difficult baby, but it is clear that I can't leave him because I am breastfeeding and when } \\
\text { he is hungry and he does not see me he panics! It has already happened that I left him } 45 \text { minutes before being able to breastfeed him. } \\
\text { Then after I arrived, I breastfed him and he looked at me and he started crying. It was as if he was still anxious because I had left him } \\
\text { and it was as if I had lost him for a little while. } \\
\text { Has your child developed any specific fears? No I don't think so. He is afraid that mom will leave. When I am in the kitchen and he is in } \\
\text { the lounge, he wants to see me. The moment that I arrive he is okay. I don't see it as strange or demanding, I think it is something } \\
\text { normal. He has to develop the capacity to play alone. Playing with his dummy is perhaps his favourite toy at this moment. Especially } \\
\text { given that he is teething. I did not see it like that before, because I did not think of a dummy as a toy, but for him it is like a toy I think. }\end{array}$ \\
\hline 7 & $\begin{array}{l}\text { He woke up one time when I had someone look after him and he was in a state of panic, he was looking for me. So I really regretted } \\
\text { having done that because his little insecurity seemed to have started there. He woke up once and I was not there. I maybe have a little } \\
\text { broken that relationship of confidence that mum is always there, don't worry. I must say that I was not happy with myself. In } \\
\text { retrospect, perhaps I should not have left when he was sleeping. From that point on, I have the impression that he wonders, "are you } \\
\text { going to be there mum?" }\end{array}$ \\
\hline
\end{tabular}

Note: Interviews originally conducted in French and have been translated to English. Dummy = pacifier. Interviewer comments in italics; participant comments in bold. 
Table 2.

Descriptive statistics for whole sample and by child sex.

\begin{tabular}{|l|r|r|r|r|r|r}
\multicolumn{1}{c}{} & \multicolumn{2}{c}{ Whole Sample } & \multicolumn{2}{c}{ Males } & \multicolumn{2}{c}{ Females } \\
\multicolumn{1}{c|}{} & \multicolumn{1}{c}{ Mean } & \multicolumn{1}{c}{ SD } & \multicolumn{1}{c}{ Mean } & \multicolumn{1}{c}{ SD } & Mean & \multicolumn{1}{c}{ SD } \\
\hline Mother's age & 30.82 & 3.997 & 30.33 & 4.254 & 31.33 & 3.680 \\
\hline Mini-PRFI & 4.46 & 1.402 & 4.58 & 1.401 & 4.33 & 1.408 \\
\hline AAI RF & 4.54 & 1.262 & 4.46 & 1.398 & 4.62 & 1.114 \\
\hline Maternal insensitivity & 2.74 & 2.466 & 2.56 & 2.510 & 2.91 & 2.438 \\
\hline
\end{tabular}


Table 3.

Zero-order correlations among key study variables.

\begin{tabular}{|c|c|c|c|c|c|c|c|}
\hline & 1 & 2 & 3 & 4 & 5 & 6 & 7 \\
\hline 1. Infant's sex ${ }^{a}$ & --- & .13 & -.09 & .05 & .07 & .12 & $.25^{*}$ \\
\hline 2. Mother's age & & --- & -.05 & .04 & -.05 & .06 & .12 \\
\hline 3. Mini-PRFI & & & --- & $.51^{* *}$ & $-.24 *$ & -.15 & $.34^{* *}$ \\
\hline 4. AAI RF & & & & --- & -.19 & -.01 & $.22^{*}$ \\
\hline 5. Maternal insensitivity & & & & & --- & $.28^{* *}$ & $.31^{* *}$ \\
\hline 6. Secure Infant Attachment & & & & & & --- & $-.52^{* * *}$ \\
\hline 7. Disorganized Infant Attachment ${ }^{b}$ & & & & & & & --- \\
\hline
\end{tabular}

${ }^{\mathrm{a}} 1=$ males, 2 = females

${ }^{\mathrm{b}} 0=$ disorganized, $1=$ organized

${ }^{\mathrm{c}} 0=$ secure, $1=$ insecure 

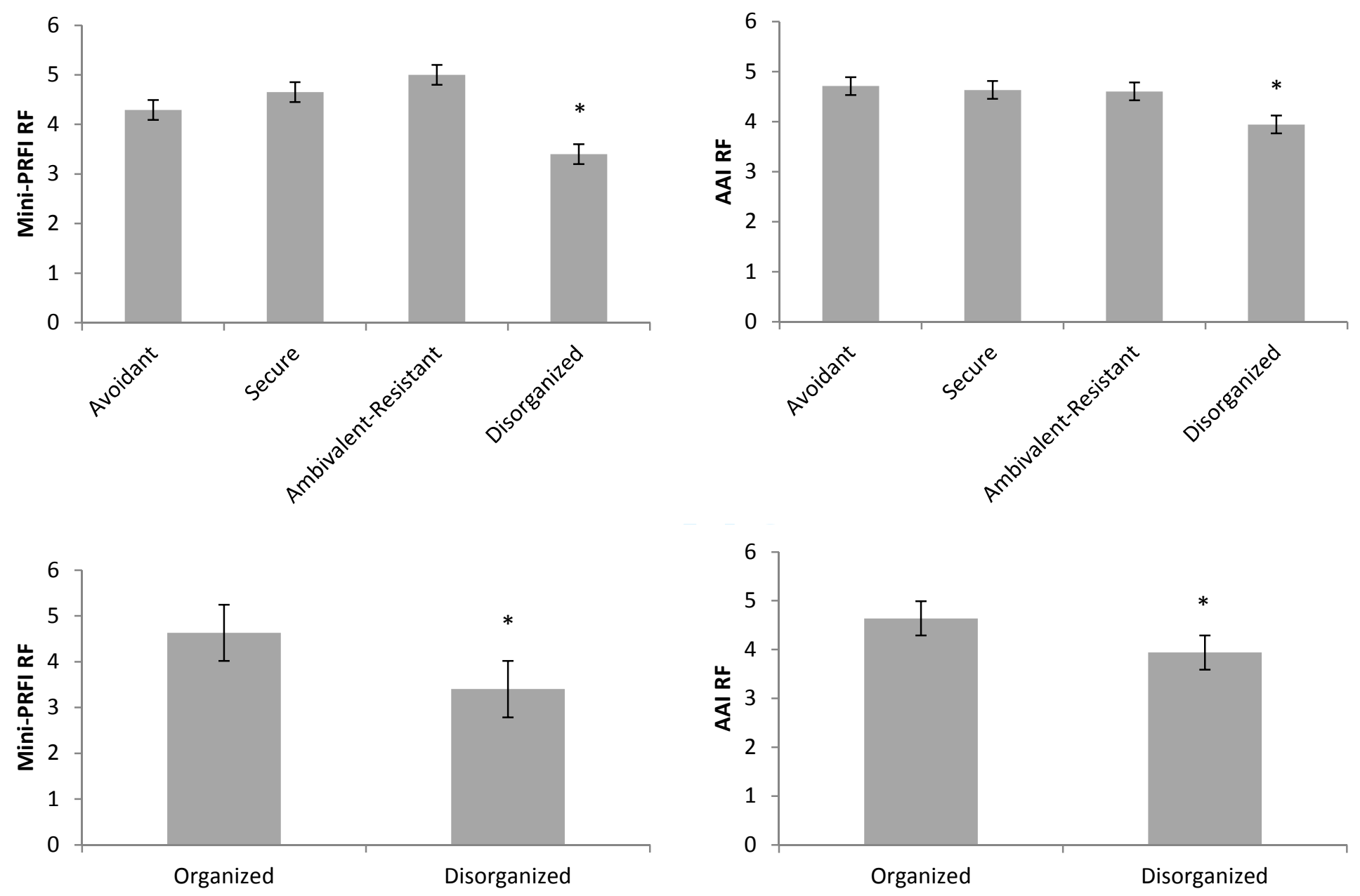

Figure 1. Mothers' RF scores on the Mini-PRFI and AAI as a function of infants' attachment classifications measured using the 4-way and 2-way categories.. 


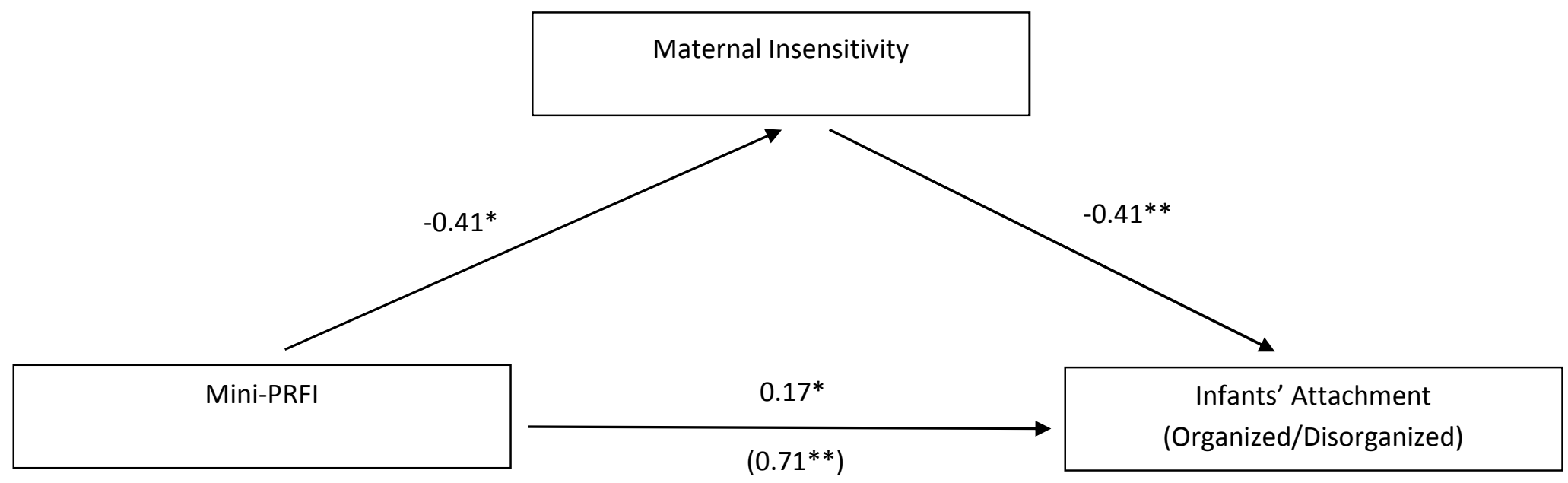

Figure 2. Mediation model of mothers' Mini-PRFI and infants' attachment classification, with maternal insensitivity as the mediating variable. Each path displays b values, with the direct (unmediated) pathway in parentheses. Children's sex controlled in analyses. Note that the infant attachment variable is a dichotomized disorganized/organized variable, with $0=$ disorganized and $1=$ organized. The values for the model predicting secure/insecure infant attachment were similar but the sign was inverted (because the variable was coded in the opposite direction, with higher scores signifying insecure attachment).

${ }^{*} p<.05, * * p<.01$. 


\section{Appendix 1. Mini PRFI for parents with 6 month old babies}

After 10 minute play sesssion

1. What are your thoughts/impression of how the play session went?

2. Do you have the impression that he played in the same way or differently from how he plays at home?

3. Could you talk to me about the play activities you typically engage in with your child at home?

4. What type of play does he prefer/like the most?

5. What is his favourite game/way of playing ?

6. How do you know when he wants to play?

7. How do you know that he wants to stop playing?

8. Have you noticed a difference in his réactions when he plays with someone else (father, grandparents)?

How do you understand that?

Temperament/Personality

1. Can you say a little bit/describe (name of the child)? How would you describe (name of child's) temperament/personality? Is there anything else you would like to add about his temperament/personality?

2. Has your child developed any specific fears? Or is there something in particular that that disturbs him in a way that is obvious?

3. Now I would like to ask you about crying? How often does he cry? Would you say he cries often ?

What makes him cry most frequently? 
When he cries, what calms him?

How long does it take to calm him?

4. When you express affection (hugs, kisses, cuddles or tickling for example), how does he react?

5. How does (child's name) react during those times when he is left on his own (for example in his cot, on his play mat or in his play pen?)

6. What about being in the arms rather than being placed in a baby seat?

7. Now I would like to ask you some questions about (child's name) and how he reacts to being taken care of by someone other than you or (other parent). First maybe you can first say something about the context and whether you have gone back to work and if so who looks after (child's name)?

Are there times when you have someone look after or babysit (child's name)? If yes can you tell me who by and and how often and how your baby reacts when he is looked after? How do you react when he is looked after by someone else?

8. Now I would like to ask you about giving (child's name) the bottle or the breast. Could you tell me a bit about whether you are breast or bottle feeding and how you made the decison around this? Have you had any difficulties around this?

In general, how do you decide to feed him or know when he is hungry? 